\title{
Impact of Learners on Emergency Medicine Attending Physician Productivity
}

\author{
Rahul Bhat, MD*† \\ Jeffrey Dubin, $\mathrm{MD}^{\dagger}$ \\ Kevin Maloy, MD ${ }^{\dagger}$
}

\author{
* Georgetown University Hospital, Department of Emergency Medicine, Washington, \\ District of Columbia \\ † Washington Hospital Center, Department of Emergency Medicine, Washington, District \\ of Columbia
}

Supervising Section Editor: Erik Barton, MD

Submission history: Submitted January 15, 2013; Revision received June 11, 2013; Accepted July 2, 2013

Electronically published September 25, 2013

Full text available through open access at http://escholarship.org/uc/uciem_westjem

DOI: 10.5811/westjem.2013.7.15882

Introduction: Several prior studies have examined the impact of learners (medical students or residents) on overall emergency department (ED) flow as well as the impact of resident training level on the number of patients seen by residents per hour. No study to date has specifically examined the impact of learners on emergency medicine (EM) attending physician productivity, with regards to patients per hour (PPH). We sought to evaluate whether learners increase, decrease, or have no effect on the productivity of EM attending physicians in a teaching program with one student or resident per attending.

Methods: This was a retrospective database review of an urban, academic tertiary care center with 3 separate teams on the acute care side of the ED. Each team was staffed with one attending physician paired with either one resident, one medical student or with no learners. All shifts from July 1, 2008 to June 30, 2010 were reviewed using an electronic database. We predefined a shift as "Resident" if > 5 patients were seen by a resident, "Medical Student" if any patients were seen by a medical student, and "No Learners" if no patients were seen by a medical student or resident. Shifts were removed from analysis if more than one learner saw patients during the shift. We further stratified resident shifts by EM training level or off-service rotator. For each type of shift, the total number of patients seen by the attending physician was then divided by 8 hours (shift duration) to arrive at number of patients per hour.

Results: We analyzed a total of 7,360 shifts with 2,778 removed due to multiple learners on a team. For the 2,199 shifts with attending physicians with no learners, the average number of PPH was $1.87(95 \%$ confidence interval $[\mathrm{Cl}] 1.86,1.89)$. For the 514 medical student shifts, the average PPH was $1.87(95 \% \mathrm{Cl} 1.84,1.90), \mathrm{p}=0.99$ compared with attending with no learner. For the 1,935 resident shifts, the average PPH was $1.99(95 \% \mathrm{Cl} 1.97,2.00)$. Compared with attending physician with no learner, attending physicians with a resident saw more PPH (1.99 vs $1.87, p<0.005)$. There was no statistically significant difference found between EM1: 1.98PPH, EM2: 1.99PPH, EM3: 1.99PPH, and off-service rotators: 1.99PPH.

Conclusion: EM attending physicians paired with a resident in a one-on-one teaching model saw statistically significantly more patients per hour ( 0.12 more patients per hour) than EM attending physicians alone. EM attending physicians paired with a medical student saw the same number of patients per hour compared with working alone. [West J Emerg Med. 2014;15(1):41-44.]

\section{INTRODUCTION}

Academic emergency departments (ED) have various staffing models for emergency medicine (EM) attending physician coverage. Those that do not alter EM attending physician coverage based on presence or absence of learners (medical students or resident physicians) may have varying 
rates of productivity when learners are present or absent. Several prior studies have examined the impact of learners on overall ED flow, as well as the impact of resident training level on the number of patients seen per hour. ${ }^{1-8}$ Four studies examined the difference in patients per hour for residents at varying post graduate training levels, and found a somewhat higher rate for each additional year of training. ${ }^{1,2,7,8}$ Other studies examined the effect of learners on overall department throughput, and while one study found that residents slowed throughput, another found that residents had no impact, while medical students did not affect throughput in two studies. ${ }^{3-6}$ No study to date has specifically examined the impact of learners on EM attending physician productivity, with regards to patients per hour (PPH). The purpose of this study was to examine whether learners are associated with an increase, decrease, or no effect on productivity of EM attending physicians.

\section{METHODS}

This was a retrospective database review examining the number of new patients seen per hour by EM attending physicians and was institutional review board approved with an exempt designation. The study setting was the main (acute care side) ED at an urban, academic, tertiary care, level 1 trauma center with a post graduate year (PGY) 1-3 residency program. Annual census during the study period was approximately 82,000 patients per year, with a $26 \%$ admission rate and about $50 \%$ of all hospital admissions coming through the ED. Nearly all patients are adults and non-trauma, as trauma patients are cared for in a separate unit and children are cared for in an adjacent children's hospital. There is also a separate fast track side of the ED for low acuity patients, with about $25 \%$ of total daily volume seen on this side.

In the main ED (acute care side), patients are sequentially assigned to one of 3 teams each led by an EM attending physician. Each team cares for patients of roughly equal acuity. We estimated that about $30 \%$ of the time, the attending physician is paired with one learner, either one resident physician (generally an EM resident or a rotating PGY-2 internal medicine or PGY-1 general surgery resident) or one fourth-year medical student. Using the Amalga electronic database (Microsoft, Redmond, WA), a query was used for each EM attending physician during the course of 2 academic years (July 1, 2008 to June 30, 2010) evaluating the total number of patients seen for each shift. Each EM attending physician's total number of patients per shift was then divided by 8 hours (the length of time during each shift when new patients are seen and evaluated) to arrive at the number of patients per hour (PPH). Each shift's calculated PPH was then averaged and categorized as EM attending with no learners, EM attending working with a resident, or EM attending working with a medical student. Medical student shift times did not always match attending and resident start/stop times resulting in shifts with multiple learners. We excluded shifts
Attending Patients Per Hour: by Team

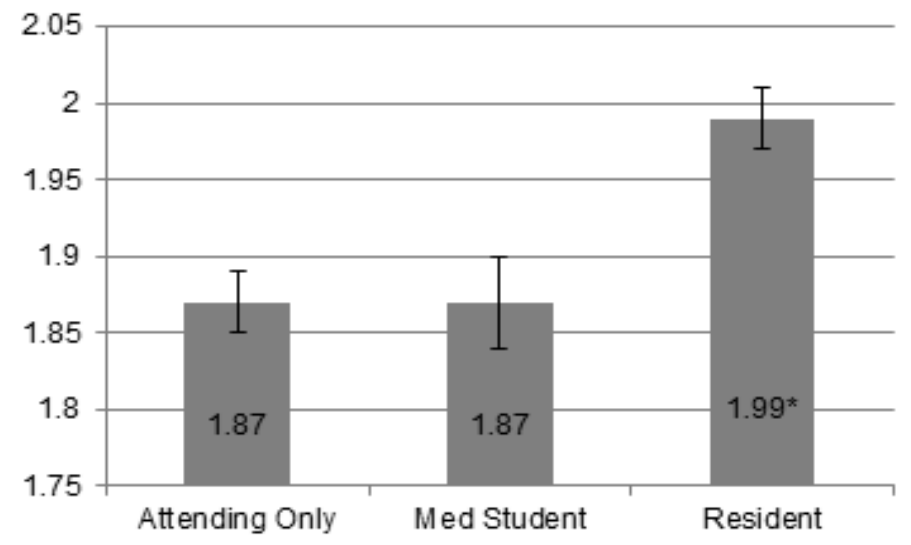

*Statistically significant

Figure 1. Attending patients per hour by team: attending, 1.87 (95\% confidence interval $[\mathrm{Cl}] 1.86,1.89)$, attending with medical student. $1.87(95 \% \mathrm{Cl} 1.84,1.90), \mathrm{p}=0.99$, and attending with resident $1.99(95 \% \mathrm{Cl} 1.97,2.00), \mathrm{p}<0.005$.

with multiple learners, as there was no way to assess the impact of each learner on attending productivity. The category of EM attending working with a resident was then further analyzed by a predetermined subgroup analysis to evaluate whether there was a significant difference in PPH when working with first-, second-, or third-year EM residents (EM1, EM-2, EM-3 respectively), as well as off-service residents.

\section{Sample Size}

To obtain adequate power to detect a difference in $\mathrm{PPH}$, we made the following assumptions: During the study period, the standard deviation of PPH in our ED was 0.28. In practice, the lowest meaningful difference in number of patients seen during a shift was 1 patient, which meant that the lowest meaningful detectable difference in PPH was 0.125 . Using an alpha of 0.05 and a power level of $80 \%$, we needed to evaluate 80 EM attending shifts working with a resident. On average, the number of acute care shifts per month per EM attending physician in our department is 12 . Based on our estimate of each attending working $30 \%$ of his/her shifts with a single learner, each attending would work 4 shifts with 1 learner per month. This meant we needed to evaluate 20 months of data to adequately power the study to have at least 80 shifts in each group. To reduce the impact of bias introduced by months with new resident physicians (July, August), we evaluated 2 entire years of data. We then compared the average PPH in each group using a two-sample, two-tailed t-test to determine significance.

\section{RESULTS}

We analyzed a total of 7,360 shifts with 2,778 removed due to the presence of multiple learners on a team. The remaining 4,582 shifts were then divided into EM attending with no learners, EM attending with a resident, or EM attending with a medical student. For the 2199 shifts with EM attending physicians with no learners, the average number of $\mathrm{PPH}$ was 
Attending Patients Per Hour: By Training level

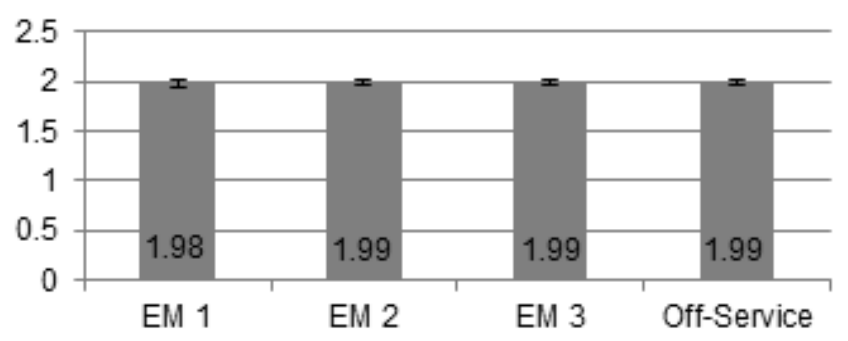

Figure 2. Attending patients per hour by resident training level, $p=0.82$.

1.87 (95\% confidence interval [CI] 1.86,1.89). For the 514 shifts with a medical student, the average attending PPH was $1.87(95 \%$ CI $1.84,1.90), p=0.99$. For the 1935 shifts with a resident, the average attending PPH was 1.99 (95\% CI 1.97,2.00), $\mathrm{p}<0.005$, which was statistically more when compared with attending physician with no learners ( Figure 1). In the subgroup analysis of EM attending with a residents of different training level, we found no statistically significant difference between EM1: 1.98, EM2: 1.99, EM3: 1.99, or offservice resident: $1.99, \mathrm{p}=0.82$ ( Figure 2).

\section{DISCUSSION}

To date, this is the first study that has specifically looked at the productivity impact of learners in a one-on-one teaching model. The average PPH seen by each solo attending physician (1.87) was somewhat lower than the 2.07 average patients per hour seen in departments with greater than 45,000 annual visits (insert additional book references here) and on the lower end of the average 1.5 to $2.5 \mathrm{PPH}$ quoted in the 2009 American College of Emergency Physicians salary survey. ${ }^{9,10}$ Likely, the reason for this difference is that patients seen in this department are adult only, with a separate fast track, leaving higher acuity patients of higher complexity seen on each team on the main side. Very few patients are seen and dispositioned prior to going to a team as there is only a limited area for triaged patients to be seen by physicians. Additionally, during the study period, our department suffered from "exit block," with $61.8 \%$ of admitted patients classified as boarding with an average of 2.8 boarding hours per admitted patient.

In our study, increasing resident training level did not contribute to more PPH for attending physicians. Prior research has indicated that higher training level is associated with greater resident $\mathrm{PPH}$; however the effect on attending PPH has not been studied. ${ }^{1,2,7,8}$ It may be that in a one-onone teaching model, despite senior residents seeing more patients than junior residents, the attending physician does not see proportionately more patients. The implications for an academic training center are unclear, as clinical productivity of attending physicians is only one of many parameters affecting attending reimbursement. The results of this study may, however, be useful in determining necessary attending coverage for staffing the department.

\section{LIMITATIONS}

The notable limitations of this study were its retrospective design and that data obtained were from a single site. In addition, we used PPH as our productivity metric instead of RVUs because our ED is largely a medical ED with few procedures on the main (acute care) side. Thus the RVU/ hour metric would reflect PPH and would not add additional information. In an ED where many procedures are done, learners may increase or decrease RVU productivity depending upon level of the learner and attending time involved to supervise the key portion of any procedure.

\section{CONCLUSION}

EM attending physicians paired with a resident in a oneon-one teaching model saw statistically significantly more patients per hour ( 0.12 more patients per hour) than EM attending physicians alone. EM attending physicians paired with a medical student saw the same number of patients per hour compared with working alone. The results of this study may help guide EDs seeking to expand or establish a residency-training program to assess the productivity impact of this decision.

Address for Correspondence: Rahul Bhat, MD. MedStar Washington Hospital Center, 110 Irving St. NW, Washington, DC 20010. Email: rgbhat77@gmail.com.

Conflicts of Interest: By the WestJEM article submission agreement, all authors are required to disclose all affiliations, funding sources and financial or management relationships that could be perceived as potential sources of bias. The authors disclosed none.

\section{REFERENCES}

1. Dowd MD, Tarantino C, Barnett TM, et al. Resident efficacy in a pediatric emergency department. Acad Emerg Med. 2005;12(12):1240-1244.

2. Thibodeau LG, Geary SP, Werter C. An evaluation of resident work profiles, attending-resident teaching interactions, and the effect of variations in emergency department volume on each. Acad Emerg Med. 2010;17 Suppl 2:S62-66.

3. Lammers RL, Roiger M, Rice L, et al. The Effect of a New Emergency Medicine Residency Program on Patient Length of Stay in a Community Hospital Emergency Department. Acad Emerg Med. 2003;10(7):725-730.

4. Chan L, Kass LE. Impact of Medical student preceptorship on ED patient throughput time. Am J Emerg Med. 1999;17:41-43.

5. McGarry J, Krall S, McLaughlin T. Impact of Resident Physicians on Emergency Department Throughput. West J Emerg Med. 2010;11(4):333-335.

6. Cobb T, Jeanmonod D, Jeanmonod R. The Impact of Working with Medical Students on Resident Productivity in the Emergency 
Department. West J Emerg Med. 2013 (in press).

7. Langdorff MI, Strange G, Macneil P. Computerized Tracking of Emergency Medicine Resident Clinical Experience. Acad Emerg Med. 1990;19(7):764-773.

8. Brennan D, Silvestri S, Sun JY, et al. Progression of emergency medicine resident productivity. Acad Emerg Med. 2007;14(9):790-794.
9. Vukmir RB, Howell RN. Emergency medicine provider efficiency: the learning curve, equilibration and point of diminishing returns. Emerg Med J. 2010;27(12):916-920.

10. American College of Emergency Physicians News. Salary Survey says Average EP Makes More than $\$ 300,000$. July 2009, Available at: http:// www.acep.org/content.aspx?id=45806. Last accessed June 11, 2013. 